\title{
Bias in memory predicts bias in estimation of future task duration
}

\author{
Michael M. RoY \\ University of Illinois at Urbana-Champaign, Urbana, Illinois \\ AND \\ Nicholas J. S. Christenfeld \\ University of California, San Diego, La Jolla, California
}

\begin{abstract}
Both anecdotal accounts and experimental evidence suggest that people underestimate how long it will take them to complete future tasks. A possible reason for this tendency is that people remember tasks as taking less time than they actually did, with these biased memories causing a corresponding bias in prediction. Two experiments were performed to determine whether or not a systematic bias in memory could explain a similar systematic bias in prediction. In support, it was found that (1) the tendency to underestimate future duration disappears when the task is novel, (2) there is similar bias in estimation of both past and future durations, and (3) variables that affect memory of duration, such as level of experience with the task and duration of delay before estimation, affect prediction of duration in the same way. It appears that, at least in part, people underestimate future event duration because they underestimate past event duration.
\end{abstract}

It often takes people longer than planned to finish tasks. Even though people are aware that their estimates have come up short in the past, they continue to underestimate the duration of future projects. Studies have shown underestimation for such tasks as writing papers, performing everyday and school tasks, completing a computer assignment (Buehler, Griffin, \& Ross, 1994; Connolly \& Dean, 1997; Griffin \& Buehler, 1999; Koole \& Spijker, 2000; Newby-Clark, Ross, Buehler, Koehler, \& Griffin, 2000; Taylor, Pham, Rivkin, \& Armor, 1998), building a computer stand (Byram, 1997), doing a spell check task (Francis-Smythe \& Robertson, 1999), completing tax forms (Buehler, Griffin, \& MacDonald, 1997), finishing Christmas shopping (Buehler \& Griffin, 2003), completing Web development projects (Moløkken \& Jørgensen, 2005), programming software (Jørgensen \& Sjøberg, 2001), reading a manuscript (Josephs \& Hahn, 1995), and waiting in line for gas (Konečni \& Ebbesen, 1976).

In the business world, underestimation of task duration has numerous negative consequences: missed deadlines, budget overruns, terminated contracts, and loss of business. These negative effects of task underestimation may be quite large in the multimillion dollar software design business (Connolly \& Dean, 1997).

The memory bias account (Roy, Christenfeld, \& McKenzie, 2005) supplies a possible explanation for this tendency to underestimate future duration: Our memories for past durations are incorrect, causing corresponding errors in prediction. People remember past task durations as being shorter than they actually were, and therefore, when they consult them to predict the next task, they underestimate how long that task will take.

Memories of past task durations will generally be based on estimates, rather than on actual measured durations, since it is rare that people know the exact beginning and ending times of tasks, especially when the task is completed over a number of different sessions. Studies of software development companies have shown that the people in charge of predicting how long projects will take rarely know the actual duration for previous, similar projects (Jørgensen \& Sjøberg, 2001; Moløkken \& Jørgensen, 2005). Furthermore, research has indicated that there is a tendency to underestimate task duration retrospectively (for reviews, see Block \& Zakay, 1997; Fraisse, 1963; Poynter, 1989; Wallace \& Rabin, 1960). If people rely on estimations and estimations tend to be short, predictions are likely to underestimate actual durations.

It is unlikely that when people predict future duration, they recall every past instance of similar tasks and estimate the duration for each. At times, people may recall the duration of a specific instance, but they may also call upon a more general representation that they have for that task that is based on past experience. This past experience could be one of performing the task directly or observing others. A prediction then could be made by adjusting this general representation of duration for a task up or down on the basis of the specific task at hand. The process of predicting task duration is similar to that of remembering

M. M. Roy, roym@etown.edu 
task duration using reconstructive memory, in which an estimation is made by calling upon a general representation for task duration and adjusting up or down, depending on memory of whether or not the task was shorter or longer than average. There is evidence that this sort of strategy is employed when people estimate the duration of past autobiographical (Burt, 1992; Burt, Kemp, \& Conway, 2001) and public (Burt \& Kemp, 1991) events. If this general representation is an underestimation of actual average duration, even when the prediction is adjusted up or down on the basis of the task at hand, the resulting prediction will underestimate task duration.

It has been found that memory reconstruction can lead to biases and errors (e.g., Vicente \& Brewer, 1993). In terms of time estimation, reconstruction can lead to underestimation in two different ways: Either people can forget certain parts of the task and, therefore, base duration on only part of the task, or people can remember all the parts of the task accurately but just remember them all as taking less time than they actually did. It appears that at least in some cases, participants remember the whole task as taking less time than it actually did. A number of experiments that have shown an overall tendency to underestimate have taken place in a lab setting, with a participant completing a task and then, right after completion, estimating overall task duration (for reviews, see Fraisse, 1963, 1984). It seems unlikely that in this case, underestimation could have been due to the participant's forgetting part of a just-completed task. There are various cognitive explanations for why, without forgetting any part of a task, people can still remember its duration as shorter than it was. For instance, it has been found that the size of memory storage for the task (Ornstein, 1969) and the number of contextual changes in a task (Block \& Reed, 1978) can cause bias in remembered duration. A task that requires only small memory storage space or has relatively few contextual changes may be remembered as being shorter than it actually was.

The idea that prediction is biased because of biased memory can be contrasted with previous explanations that have been centered on the idea that prediction is flawed because people are overly optimistic and underutilize memory for how long similar tasks have taken in the past. A number of studies have examined possible methods by which to correct prediction by having participants utilize information that they have purportedly previously ignored during prediction. To this end, participants have been made aware of possible surprises that could arise during the task (Byram, 1997; Hinds, 1999), have broken tasks down into their individual components (Byram, 1997; Connolly \& Dean, 1997), have examined the information as observers instead of as actors (Buehler et al., 1994; Byram, 1997; Hinds, 1999; Newby-Clark et al., 2000), have formed alternative scenarios of how the task could be completed (Byram, 1997; Newby-Clark et al., 2000), and have recounted past completion times (Buehler et al., 1994; Hinds, 1999). These methods, for the most part, have been unsuccessful at increasing the overall accuracy of prediction. The participants continued to be biased in their estimates of the durations of future events, even though past memories were made salient during prediction.
These ideas-bias being due to an optimistic outlook and to ignoring memory for the duration of similar past tasks - are central to the planning fallacy theory (Buehler, Griffin, \& Ross, 2002; Kahneman \& Tversky, 1979, 1982), which is an attempt to explain why people continue to underestimate future task durations even though they are aware of having failed to meet their expectations in the past. It has been proposed that when making a prediction, people focus narrowly on the task at hand and form an overly optimistic scenario that ignores memories of when similar tasks have been finished previously. Therefore, Kahneman and Tversky proposed that prediction will be best if it is based on memory for how long similar tasks have taken in the past.

The planning fallacy and other theories hold that people have memories available that could improve prediction but that people fail to utilize them. In contrast, the memory bias theory holds that memory is consulted during prediction, with bias occurring because memory for past duration is incorrect. In an earlier review article (Roy et al., 2005), we discussed areas in which these two accounts predict differences in time estimation behavior. Here, we set out to test empirically, with two experiments, three areas in which the theories predict different results.

\section{EXPERIMENT 1}

If the tendency to underestimate future duration is due to biased memory, underestimation should be most likely for tasks that are familiar. It is less likely that novel tasks should be reliably underestimated; it may be that novel tasks are overestimated or that there is more overall error in prediction. In contrast, it may be expected that if underestimation is due to memory's not being consulted, underestimation will be greatest for novel tasks. If there is a tendency to form overly optimistic scenarios of how a task will be finished and to disregard memories of past completion times, this tendency should be greatest when there are no memories of past duration at our disposal. With nothing to ground prediction in reality, the effect of being overly optimistic should be greatest. Kahneman and Tversky $(1979,1982)$, in their discussion of the planning fallacy, stated that any factor that increases the "perceived uniqueness" of the task should increase the tendency to underestimate.

In support of the memory bias account, one experiment showed that participants were more likely to overestimate the duration it would take to play a piece of music when it was novel but underestimated when it was very familiar (Boltz, Kupperman, \& Dunne, 1998). In Experiment 1, we sought to replicate this result and to compare past and future estimations of duration for a novel task. Memory bias predicts that there should be a difference between estimations of duration given before and after the task. Previous research has indicated that participants estimating how long the task will take should overestimate when the task is novel. Participants who estimate duration after performing a novel task are more familiar with the task than are participants estimating before the task. Their experience with the task should increase the tendency to underestimate the duration (Boltz et al., 1998; Hinds, 1999). 
$H_{1}$ : Prediction of novel task duration is less likely to be an underestimation than is memory of task duration.

In Experiment 1, we examined whether there are differences between estimations made either before or after participants constructed three origami rabbits. ${ }^{1}$ Estimates were compared with the actual durations.

\section{Method}

Participants. Forty-five University of California, San Diego, students ( 27 of them female, 18 male) participated. The participants received course credit for their psychology classes in exchange for participation.

Procedure. The participants arrived singly for the experiment and were asked by the experimenter to remove their watches and any rings or bracelets they were wearing. They were told that this was because they would be working with their hands on the task, although it actually served to ensure that they could not consult their watches and calculate how long the task was taking. There was also no clock in the room. The participants were informed that they would be making three origami rabbits. They were given diagrammatic instructions on how to make the rabbits, a sample rabbit, three blank $8.5 \times 8.5$ in. sheets of paper, a pair of scissors, and a pen. The participants were randomly assigned to estimate duration before or after the task. After looking over the instructions, the 23 participants in the future condition were asked to estimate, in minutes and seconds, how long the task would take and then were asked to perform the task. The 22 participants in the past condition examined the instructions and then performed the task, after which they were instructed to estimate how long it had taken them. The experimenter surreptitiously timed the participants during the task.

Dependent measure. In order to assess accuracy of estimates, an index was created by taking the log of the ratio of estimated duration to actual duration. This index, which we will refer to as log proportional error, has a number of benefits. First, it simplifies interpretation of the results: A negative score indicates underestimation, a score of zero indicates perfect accuracy, and a positive score indicates overestimation. Second, by converting to proportional bias, it makes it possible to directly compare differences between estimated and actual durations for tasks of varying duration. Third, the index normalized the data; there was a strong positive skew in estimates of duration.

\section{Results}

The participants in the past condition underestimated how long it had taken them to complete the task [mean $\log$ proportional error $=-.154$, significantly different from $0 ; t(21)=-3.34, p=.003, d=0.71$ ], estimating that it took them 10.0 min (median) to make the rabbits, whereas it actually took them $13.5 \mathrm{~min}$. In contrast, the participants in the future condition significantly overestimated how long it would take to complete the task [mean $\log$ proportional error $=.096 ; t(22)=4.11, p=.0005$, $d=0.86]$, predicting that it would take them $20.0 \mathrm{~min}$ to complete the task, even though it actually took them $11.0 \mathrm{~min}$. There was a significant difference between the participants who estimated duration before the task and those who estimated duration after the task $[t(43)=4.89$, $p<.0001, d=1.45]$.

Although it took the participants in the past condition slightly longer to complete the task than it took the participants in the future condition, the difference was not significant $[t(43)=-.93, p=.36]$.

\section{Discussion}

Consistent with previous research on retrospective estimation of duration (Block \& Zakay, 1997; Fraisse, 1963;
Poynter, 1989; Wallace \& Rabin, 1960), the results indicate that there was a tendency to underestimate past task duration. However, replicating previous results (Boltz et al., 1998), future task duration was overestimated when the task was novel. This is in contrast to the results in the majority of previous research, which has shown an overall tendency to underestimate future task duration (Buehler \& Griffin, 2003; Buehler et al., 1997; Buehler et al., 1994; Byram, 1997; Connolly \& Dean, 1997; Francis-Smythe \& Robertson, 1999; Griffin \& Buehler, 1999; Jørgensen \& Sjøberg, 2001; Josephs \& Hahn, 1995; Konečni \& Ebbesen, 1976; Koole \& Spijker, 2000; Moløkken \& Jørgensen, 2005; Newby-Clark et al., 2000; Taylor et al., 1998). If the participants had no memory of relevant past task durations, the tendency to underestimate a future task disappeared.

There was a difference in bias between memory and prediction for the novel task. Whereas the participants unfamiliar with the task overestimated how long it would take, those who performed the task underestimated how long it took. If bias in prediction is due to bias in memory, participants who underestimated how long the task took should, after having done it, underestimate how long it will take to perform the task again. In the next experiment, we examined whether or not giving participants experience with a task would change bias in prediction from overestimation to underestimation.

Although not significant, there was a difference in time to complete the task for the participants in the past and the future conditions, with the participants performing the task slightly quicker when a prediction was made before performing the task. It is possible that even though the participants in the past and the future conditions performed the same task, the difference in their performance of that task may have been due to when the estimation was given. The act of making a prediction can be a form of goal setting and may result in a change in behavior (Locke \& Latham, 1990). The participants in the future condition, unlike the participants in the past condition, were aware, while performing the task, that its duration was important to the experiment. So even though using the same task makes it easier to compare between groups, it must be acknowledged that there are possible differences in performance on the task due to when estimation was given.

\section{EXPERIMENT 2}

If prediction of future duration is based on memory of previous task experience, the overall bias in prediction and memory should be similar. Given an overall tendency to underestimate task duration in retrospect, there should be a similar tendency to underestimate how long the task will take in the future. In contrast, if bias is due to memory's not being consulted during prediction, it would be expected that memory would be more accurate than prediction. One could not reasonably blame prediction shortcomings on a failure to consult memories of past task experiences if those memories are equally biased.

Previous research has indicated that there is a similar tendency for both remembered duration and predicted du- 
ration to be an underestimation of actual duration. However, these studies showing an overall tendency for underestimation for both memory and prediction have differed in too many ways - task, method, and participants - to allow direct comparison and confident conclusions. In Experiment 2, we examined whether or not bias would be similar, in terms of direction and size, for remembered and predicted durations of a task.

$\mathrm{H}_{2}$ : There is a similar bias in both prediction and memory.

If bias in prediction is due to bias in memory, it follows that variables that affect memory should have the same effect on prediction. If, for instance, a certain condition causes people to overestimate duration in retrospect, the same condition should cause people to overestimate how long that same task will take in the future. If, on the other hand, memory is not consulted during prediction, variables that affect memory should not necessarily affect prediction.

In Experiment 2, we also examined whether or not level of experience with a task and duration of delay after the task would have the same effect on memory and prediction. Increased experience with a task has been found to increase the tendency to underestimate duration retrospectively (Boltz et al., 1998; Hinds, 1999). In a similar manner, it has been found that increasing the length of delay before past task duration is estimated can increase the amount of underestimation (Pedri \& Hesketh, 1993), although not all studies have shown this effect (Burt, 1992; Burt et al., 2001). If experience and delay affect memory of duration, they should also affect prediction of duration.

$\mathrm{H}_{3}$ : Variables that affect memory have a similar effect on prediction.

Experiment 2 focused on the effect of learning and delay on both past and future estimation for an origami task. The experiment was a $3 \times 2 \times 2$ design with level of experience with the task (one, three, or nine rabbits), delay (0 or $10 \mathrm{~min}$ ), and perspective of estimation (past or future) varied.

\footnotetext{
Method

Participants. Five hundred forty-four University of California, San Diego, students (390 of them female, 154 male) participated. The participants received course credit for their psychology classes in exchange for participation.

Procedure. The participants arrived singly for the experiment and were asked to remove their watches and any rings or bracelets they were wearing. For the low-, medium-, and high-experience conditions, the participants were instructed that they would be making one, three, or nine origami rabbits, respectively. The participants were given the instructions for making the origami rabbits, a sample rabbit, squares of blank paper, scissors, and a pen. They were given time to look over the directions and the sample and were asked to begin when they were ready. The experimenter surreptitiously timed the participants.

The participants in the past condition were asked to estimate, in minutes and seconds, how long it had taken to make the one, three, or nine rabbits either immediately after finishing the task or after a 10-min delay. In the delay condition, the participants performed three tasks that were unrelated to the estimation task - reading the Gettysburg Address, rating pictures for emotional content, and reading the Reverend Martin Luther King Jr.'s "I Have a Dream" speech - until $10 \mathrm{~min}$ had elapsed, before making their estimate.

After making one, three, or nine origami rabbits, the participants in the future condition were asked either immediately or after a
}

10-min delay (using the same distraction tasks) to estimate how long it would take them to make three more origami rabbits. They then made three more rabbits while they were timed surreptitiously.

\section{Results}

It took the participants in the low-experience condition $4.1 \mathrm{~min}$ (past, 4.2; future, 3.9) to make one rabbit, those in the medium-experience condition $9.1 \mathrm{~min}$ (past, 9.1; future, 9.2) to make three rabbits, and those in the high-experience condition 18.0 min (past, 17.6; future, 18.3) to make nine rabbits (median). As a check to the effectiveness of the level-of-experience manipulation, we examined the duration that it took participants in the future condition to make three more rabbits. The participants did improve with added practice $[F(2,316)=62.27$, $\left.p<.0001, \eta^{2}=.28\right]$, with the participants in the low-, medium-, and high-experience conditions taking 5.5, 4.6, and $3.5 \mathrm{~min}$, respectively (median). ${ }^{2}$

First, we examined whether or not there was a similar bias in memory and prediction. Recall that in Experiment 1 , the participants unfamiliar with the task predicted that it would take longer than it did, whereas the participants estimating after completing the task thought that it had taken less time than it actually did. Memory bias predicts that the participants who underestimated how long the task took should now also underestimate how long it will take in the future. Therefore, we compared the participants who had made three rabbits and had estimated how long it had taken with those who had made three rabbits and had estimated how long it would take to make three more. There was no difference in bias for prediction and memory $[t(185)=1.14, p=.25, d=0.096]$ for these groups, with both tending to underestimate task duration (mean $\log$ proportional error $=-.06$ for memory and -.03 for prediction). In comparison with Experiment 1, gaining experience with the task caused the participants to now underestimate how long the task would take.

Initially, comparison was limited to the conditions in which three rabbits were made because it allowed for the evaluation of tasks with the same level of experience and with roughly the same duration. Comparison was more difficult when all the levels of experience were included; the participants in the past condition were estimating how long it took to make one, three, or nine rabbits, whereas all the participants in the future condition were estimating how long it would take to make three rabbits. Whereas all of the participants in the future condition were making estimations for the same task, the participants in the past condition were making estimations for tasks that varied greatly in duration. When all the conditions were taken together and analyzed in a $3 \times 2 \times 2$ ANOVA, there was a difference in estimation due to perspective of estimation, past or future $\left[F(1,532)=14.24, p=.0002, \eta^{2}=.024\right]$, with a greater tendency to underestimate the duration of the task when it was in the past (mean log proportional error $=-.072$ ) than when it was in the future (mean $\log$ proportional error $=$ -.012). The difference between future and past estimation can be explained, at least partially, by differences in task duration. Regardless, the difference found here between prediction and memory was the opposite of what would be 
expected if bias in prediction was due to the disregarding of memory; memory was less accurate than prediction. At the very least, it seems clear that properly utilizing memory will not increase the accuracy of prediction.

We also examined whether or not variables that affect memory for task duration would have the same effect on prediction of duration. If either the level of experience or the duration of delay differentially affected memory and prediction, we would expect a significant interaction with perspective of estimation. For instance, we might expect that if memory and prediction are not linked, experience with the task might affect memory for how long the task took in the past, but not prediction for how long it would take in the future. On the other hand, if memory and prediction are linked, we should see a similar effect of a variable on both prediction and memory. When log proportional error was analyzed in a $3 \times 2 \times 2$ ANOVA, there were no significant interactions between perspective of estimation and experience or perspective and delay (both $F$ s $<1)$. There were, however, significant main effects of both experience and delay, affecting bias in memory for past task duration and bias in prediction for future task duration in the same way (see Figure 1). Estimation of duration moved from overestimation to underestimation as experience with the task grew $[F(2,532)=21.95$, $\left.p<.0001, \eta^{2}=.073\right]$. Mean $\log$ proportional error was .033 for the participants in the low-experience condition, -.040 for those in the medium-experience condition, and -.107 for those in the high-experience condition (a positive score indicates overestimation, and a negative index indicates underestimation). Underestimation was greater after a $10-\mathrm{min}$ delay $\left[F(1,532)=4.09, p=.04, \eta^{2}=\right.$ $.007]$, with mean $\log$ proportional errors of -.019 for no delay and -.052 after a 10-min delay.

Although the experts were more likely to underestimate task duration, they were more consistent in their predictions of future task duration. There was a decrease in overall error with greater experience, as measured by the absolute value of the estimated duration minus the actual duration $\left[F(2,313)=9.54, p<.0001, \eta^{2}=.007\right]$. Average unsigned error was $2.9 \mathrm{~min}$ for novices, $1.9 \mathrm{~min}$ for intermediates, and $1.4 \mathrm{~min}$ for experts.

\section{Discussion}

In Experiment 1, there was a difference in bias for estimations given before and after a novel task. The results for Experiment 2 indicate that supplying experience with the task before prediction eliminates this difference, with bias in memory and prediction similar in direction and size.

Variables that affected memory had a similar effect on prediction. Underestimation was greatest with more experience with the task and after a 10-min delay, whether estimation was for how long the task had taken or how long it was about to take.

As in previous studies (Boltz et al., 1998; Hinds, 1999), it was found that as experience with the task grew, estimation of duration moved from overestimation to underestimation. Experience with a task made it more likely that the task would be underestimated for both memory of past duration and prediction of future duration.

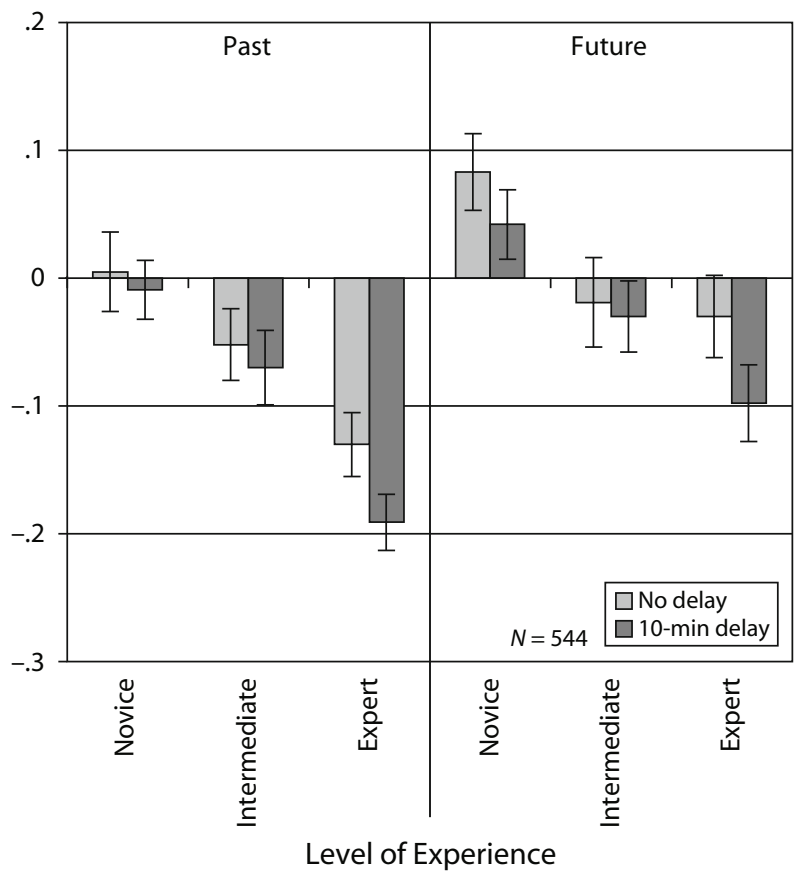

Figure 1. Log proportional error as a function of level of experience with the task (one, three, or nine origami rabbits), delay ( 0 or $10 \mathrm{~min}$ ), and perspective of estimation (past or future).

Although a significant effect of duration of delay was found, so that underestimation was greatest after a 10-min delay, these results must be interpreted with some caution. The effect size for duration of delay was very small $\left(\eta^{2}=.007\right.$; Cohen, 1988), especially in comparison with the effect size for level of experience $\left(\eta^{2}=.073\right)$. The small effect size indicates that although there may be a significant difference in estimation due to duration of delay, this difference may be negligible in terms of realworld applications. It may be that there was a small effect because $10 \mathrm{~min}$ is a short delay, although we are not able to determine with these results what the effect of a delay in terms of days or even weeks might be. It may be that with a greater delay, there would be a much larger effect than that found here.

\section{GENERAL DISCUSSION}

Predictions of future task duration are often incorrect. The memory bias account (Roy et al., 2005) supplies a possible reason: Error in memory leads to error in prediction. In support of the memory bias account, the present results indicate that bias in memory and prediction are similar when the task is familiar, but not when the task is novel, and that variables that affect memory have a similar effect on prediction of future duration. These findings are in contrast to what would have been expected if underestimation of duration were due to the improper use of memory of past duration.

Although it is possible that memory of duration and prediction of duration are completely separate processes that are coincidentally affected similarly by experience with the 
task and duration of the delay, it is more parsimonious to view past and future estimation as linked. People's memories are affected by these variables, and this is reflected in predictions of duration based on these memories.

The time estimation bias found in these two experiments is not likely caused by participants' forgetting subcomponents of the task or by their disregarding the possibility of surprises or interruptions. The task was short and simple, lasting only a few minutes. Since estimation was made right after the participants had either examined the instructions or performed the task itself, it is unlikely that in that short space, they forgot about certain aspects of the task, such as folding the ears. Also, the task was performed in a controlled environment in which surprises and interruptions were not possible. The participants continued to be biased in their estimates even though these factors were controlled.

Being overly optimistic, ignoring the fact that surprises or interruptions may happen, or forgetting about certain subcomponents of the task when making predictions may still play a role in the biasing of predictions. It is likely that at times, all of these factors influence estimation of task duration. These factors may combine with memory bias to increase the tendency to underestimate. However, these mechanisms are not necessary for underestimation to be found.

\section{Possible Reasons for a Bias in Memory}

Although it appears that bias in prediction may be caused by a similar bias in memory, it is not clear why memory is biased. A number of different theories can explain the shift in bias from overestimation to underestimation as experience with a task increases. Here, we will review some of these possible explanations for the effect of experience on bias in memory.

The shift in bias, from overestimation to underestimation as experience with the task increases, could be explained in terms of changes in the number of memories associated with a task. Memory models of times estimation hold that the smaller the memory storage size (Ornstein, 1969) or the fewer contextual changes in a task (Block \& Reed, 1978), the more likely people are to underestimate task duration. There is evidence that as people become more experienced with a task, memories for the different portions of the task are consolidated into fewer and larger "chunks" (e.g., Chase \& Simon, 1973). This consolidation of steps with greater experience would decrease memory load and, therefore, decrease memory for task duration.

Shift in bias may also be due to a change in the amount of attention that is needed to perform a task as experience with the task grows. When the task is novel, people may have to pay more attention to their performance than when the task is well learned. There is evidence that shifting the amount of attention focused on a task has differential effects on task performance for novices and experts (Beilock, Bertenthal, McCoy, \& Carr, 2004). In terms of performance, novices benefit from focusing more attention on the task, whereas experts benefit from focusing less attention on the task. It has also been found that the amount of attention focused on performing a task can affect the perceived duration of the task, with greater attention to the task leading to underestimation (Thomas \& Weaver, 1975). However, this predicts that novel tasks should be underestimated, the opposite of the results found here. We may have found a result opposite to what is predicted because attentional models of time estimation, such as those developed by Thomas and Weaver, were formed to explain estimation of very short durations (less than $1 \mathrm{sec}$ ) and may be better suited to explain the perception of passing time than to explain memory for past task duration (Block \& Zakay, 1997).

It is possible that there may be a shift in the amount of self-regulation that is needed to perform a task as the task becomes more familiar. A novel task may require people to work harder to block out possible distractions while performing the task than they would if the task was very familiar. There is evidence that increased self-regulation while performing a task - for instance, trying not to think about white elephants - will lead to longer task estimations (Vohs \& Schmeichel, 2003). Therefore, overestimation may occur for novel tasks because performing these tasks requires greater effort in blocking out distractions.

Ease of recall may also affect estimation of task duration, with quick recall associated with shorter remembered task duration (Sanna \& Schwarz, 2004). It could be that when a task is well learned, memories of that task are easier to recall and that ease of recall causes underestimation.

The bias found in prediction could be due to bounding and rounding when estimations are made (Huttenlocher, Hedges, \& Bradburn, 1990). Bounding is the tendency to form an upper boundary for how long a task could take. For instance, participants performing the origami task might use the fact that the experiment could take no longer than an hour to set an upper bound for the task. This upper bound would be too high, causing an overall tendency to overestimate task duration. Experience with the task could cause a shift in the upper boundary, moving from too large to possibly too small. An overall tendency to underestimate duration may also be caused by rounding, the tendency to gravitate toward certain values when making an estimation. If the distance between these rounded values increases as duration increases, this would lead to a greater tendency to round downward, creating an overall tendency to underestimate duration. We did find a tendency for the participants to round to certain values; in Experiment 1, a number of participants indicated that the task would take exactly 20 min when estimating before performing the task and exactly 10 min when estimating after performing the task. Bounding could help explain the shift in bias due to familiarity with the task, whereas rounding could help explain an overall tendency to underestimate.

The tendency for underestimation to increase as participants become more experienced with the task could also be explained by a tendency to overestimate how much they have improved on the task. In general, people want to view themselves in a positive light and as always improving (Ross \& Wilson, 2003). In terms of memory for task duration, after performing the task numerous times, people 
might imagine that they have made greater improvements in performance of the task than they actually have.

Finally, it is possible that totally different cognitive strategies are used to estimate duration when the task is novel and when it is familiar (Jones \& Boltz, 1989). Future research should be focused on determining which, if any, of these possible explanations can best account for the shift in bias as experience with a task changes.

\section{Implications and Summary}

At least in the area of software design, prediction for how long a project should take is usually made by an expert (Jørgensen, 2004a). However, the results from Experiment 2, along with those of previous experiments (Boltz et al., 1998; Hinds, 1999), indicate that underestimation is most likely when a task is well learned. Experience with a task seems to negatively affect predictive ability. The person who would seem to have the best ability to predict - an expert - is also the person who is most likely to be biased. Even though they are likely to be biased, there still may be valuable information in the predictions of experts. Experts may be more consistent in their predictions, just consistently biased.

The results indicate that we may be better able to understand how and why prediction will be biased if we first examine bias in memory. If a certain variable causes someone to remember a task as taking less time than it actually did, it is likely that the same variable will cause that person to underestimate how long that task will take in the future.

A number of previous studies have tried to improve predictive accuracy with little overall success. The results here offer a different possible solution: correct memory of past duration. If bias in prediction is caused by a bias in memory, a possible method for correcting prediction is to update memory. In support, a study has shown that when software designers were predicting how long a project would take, prediction was more accurate if the designers could identify previous projects that were similar and determine the duration of those projects (Jørgensen, 2004b). Given that it is rare that people know the exact beginning and ending times for a task, a possible way to correct memory is to keep detailed records of the time spent on a task. Future work should examine the efficacy of correcting memory for improving prediction.

In sum, there is a similar bias in memory of past task duration and prediction of future task duration. However, this similarity disappears when the task is novel or there is no memory to call upon for prediction. These results indicate that biased memory is a plausible cause of biased prediction of duration.

\section{AUTHOR NOTE}

Part of this work was supported by NIMH National Research Service Award MH14257 to the University of Illinois. This work was completed while the first author was a postdoctoral trainee in the Quantitative Methods Program of the Department of Psychology, University of Illinois at Urbana-Champaign. We thank Richard Block and Christopher Burt for their helpful comments in review of this article. Correspondence concerning this article should be addressed to M. M. Roy, Department of Psychology, Elizabethtown College, One Alpha Drive, Elizabethtown, PA 17022 (e-mail: roym@etown.edu).

\section{REFERENCES}

Beilock, S. L., Bertenthal, B. I., McCoy, A. M., \& Carr, T. H. (2004). Haste does not always make waste: Expertise, direction of attention, and speed versus accuracy in performing sensorimotor skills. Psychonomic Bulletin \& Review, 11, 373-379.

Block, R. A., \& ReEd, M. A. (1978). Remembered duration: Evidence for a contextual-change hypothesis. Journal of Experimental Psychology: Human Learning \& Memory, 4, 656-665.

Block, R. A., \& ZaKaY, D. (1997). Prospective and retrospective durations judgments: A meta-analytic review. Psychonomic Bulletin \& Review, 4, 184-197.

Boltz, M. G., Kupperman, C., \& Dunne, J. (1998). The role of learning in remembered duration. Memory \& Cognition, 26, 903-921.

BueHLER, R., \& Griffin, D. (2003). Planning, personality, and prediction: The role of future focus in optimistic time predictions. Organizational Behavior \& Human Decision Processes, 92, 80-90.

Buehler, R., Griffin, D., \& MacDonald, H. (1997). The role of motivated reasoning in optimistic time predictions. Personality \& Social Psychology Bulletin, 23, 238-247.

Buehler, R., Griffin, D., \& Ross, M. (1994). Exploring the "planning fallacy": Why people underestimate their task completion times. Journal of Personality \& Social Psychology, 67, 366-381.

Buehler, R., Griffin, D., \& Ross, M. (2002). Inside the planning fallacy: The causes and consequences of optimistic time predictions. In T. D. Gilovich, D. W. Griffin, \& D. Kahneman (Eds.), Heuristics and biases: The psychology of intuitive judgment (pp. 250-270). New York: Cambridge University Press.

BURT, C. D. B. (1992). Reconstruction of the duration of autobiographical events. Memory \& Cognition, 20, 124-132.

Burt, C. D. B., \& KemP, S. (1991). Retrospective duration estimation of public events. Memory \& Cognition, 19, 252-262.

BurT, C. D. B., KemP, S., \& Conway, M. (2001). What happens if you retest autobiographical memory 10 years on? Memory \& Cognition, 29, 127-136.

Byram, S. J. (1997). Cognitive and motivational factors influencing time prediction. Journal of Experimental Psychology: Applied, 3, 216-239.

Chase, W. G., \& Simon, H. A. (1973). The mind's eye in chess. In W. G. Chase (Ed.), Visual information processing (pp. 215-281). New York: Academic Press.

COHEN, J. (1988). Statistical power analysis for the behavioral sciences (2nd ed.). Hillsdale, NJ: Erlbaum.

Connolly, T., \& Dean, D. (1997). Decomposed versus holistic estimates of effort required for software writing tasks. Management Science, 43, 1029-1045.

Fraisse, P. (1963). The psychology of time (J. Leith, Trans.). New York: Harper \& Row.

FraIsSE, P. (1984). Perception and estimation of time. Annual Review of Psychology, 35, 1-36.

Francis-Smythe, J. A., \& Robertson, I. T. (1999). On the relationship between time management and time estimation. British Journal of Psychology, 90, 333-347.

GrifFIN, D., \& BUEHLER, R. (1999). Frequency, probability, and prediction: Easy solutions to cognitive illusions? Cognitive Psychology, 38, $48-78$.

HinDS, P. J. (1999). The curse of expertise: The effects of expertise and debiasing methods on predictions of novice performance. Journal of Experimental Psychology: Applied, 5, 205-221.

Huttenlocher, J., Hedges, L. V., \& Bradburn, N. M. (1990). Reports of elapsed time: Bounding and rounding processes in estimation. Journal of Experimental Psychology: Learning, Memory, \& Cognition, 16, 196-213.

Jones, M. R., \& Boltz, M. (1989). Dynamic attending and responses to time. Psychological Review, 96, 459-491.

JøRGENSEN, M. (2004a). A review of studies on expert estimation of software development effort. Journal of Systems \& Software, 70, 37-60.

JøRGENSEN, M. (2004b). Top-down and bottom-up expert estimation of software development effort. Information \& Software Technology, 46, 3-16.

JøRGENSEN, M., \& SJøвERG, D. I. K. (2001). Impact of effort estimates on software project work. Information \& Software Technology, 43, 939-948. 
Josephs, R. A., \& Hahn, E. D. (1995). Bias and accuracy in estimates of task duration. Organizational Behavior \& Human Decision Processes, 61, 202-213.

Kahneman, D., \& TVERSKY, A. (1979). Intuitive prediction: Biases and corrective procedures. TIMS Studies in Management Sciences, 12, 313-327.

Kahneman, D., \& Tversky, A. (1982). Intuitive prediction: Biases and corrective procedures. In D. Kahneman, P. Slovic, \& A. Tversky (Eds.), Judgments under uncertainty: Heuristics and biases (pp. 414 421). Cambridge: Cambridge University Press.

KoneC̆NI, V. J., \& EBBESEN, E. B. (1976). Distortions of estimates of numerousness and waiting time. Journal of Social Psychology, 100, 45-50.

Koole, S., \& SPIJKer, M. (2000). Overcoming the planning fallacy through willpower: Effects of implementation intentions on actual and predicted task-completion times. European Journal of Social Psychology, 30, 873-888.

Locke, E. A., \& LAtham, G. P. (1990). A theory of goal setting and task performance. Englewood Cliffs, NJ: Prentice Hall.

MoløKKen, K., \& JøRGensen, M. (2005). Expert estimation of Webdevelopment projects: Are software professionals in technical roles more optimistic than those in non-technical roles? Empirical Software Engineering, 10, 7-29.

Newby-Clark, I. R., Ross, M., Buehler, R., Koehler, D. J., \& GrIfFIN, D. (2000). People focus on optimistic scenarios and disregard pessimistic scenarios while predicting task completion times. Journal of Experimental Psychology: Applied, 6, 171-182.

ORnsteIn, R. E. (1969). On the experience of time. Hammondsworth U.K.: Penguin.

Pedri, S., \& Hesketh, B. (1993). Time perception: Effects of task speed and delay. Perceptual \& Motor Skills, 76, 599-608.

Poynter, D. (1989). Judging the duration of time intervals: A process of remembering segments of experience. In I. Levin \& D. Zakay (Eds.), Time and human cognition: A life-span perspective (pp. 305-322). Amsterdam: North-Holland.
Ross, M., \& Wilson A. E. (2003). Autobiographical memory and conceptions of self: Getting better all the time. Current Directions in Psychological Science, 12, 66-69.

Roy, M. M., Christenfeld, N. J. S., \& McKenzie, C. R. M. (2005). Underestimating the duration of future events: Memory incorrectly used or memory bias? Psychological Bulletin, 131, 738-756.

SANNA, L. J., \& Schwarz, N. (2004). Integrating temporal biases: The interplay of focal thoughts and accessibility experiences. Psychological Science, 15, 474-481.

Taylor, S. E., Pham, L. B., Rivkin, I. D., \& Armor, D. A. (1998). Harnessing the imagination: Mental stimulation, self-regulation, and coping. American Psychologist, 53, 429-439.

Thomas, E. A. C., \& Weaver, W. B. (1975). Cognitive processing and time perception. Perception \& Psychophysics, 17, 363-367.

Vicente, K. J., \& BREWER, W. F. (1993). Reconstructive remembering of the scientific literature. Cognition, 46, 101-128.

Vohs, K. D., \& Schmeichel, B. J. (2003). Self-regulation and the extended now: Controlling the self alters the subjective experience of time. Journal of Personality \& Social Psychology, 85, 217-230.

Wallace, M., \& Rabin, A. I. (1960). Temporal experience. Psychological Bulletin, 57, 213-236.

\section{NOTES}

1. A separate sample of University of California, San Diego $(n=99)$ students drawn from the same participant pool indicated that the majority of them $(77 \%)$ had little or no previous experience in constructing origami objects. It is likely that even fewer had experience with origami rabbits.

2. ANOVA on log of actual duration due to positive skew in data.

(Manuscript received March 2, 2005; revision accepted for publication January 12, 2006.) 